\title{
Linear and nonlinear ion-acoustic waves in nonrelativistic quantum plasmas with arbitrary degeneracy
}

\author{
Fernando Haas \\ Physics Institute, Federal University of Rio Grande do Sul, CEP 91501-970, Avenida Bento Gonçalves 9500, Porto Alegre, RS, Brazil \\ Shahzad Mahmood \\ Physics Institute, Federal University of Rio Grande do Sul, CEP 91501-970, Avenida Bento Gonçalves 9500, Porto Alegre, RS, Brazil \\ and Theoretical Physics Division (TPD), PINSTECH, P. O. Nilore, Islamabad 44000, Pakistan
}

(Received 1 June 2015; revised manuscript received 3 November 2015; published 25 November 2015)

\begin{abstract}
Linear and nonlinear ion-acoustic waves are studied in a fluid model for nonrelativistic, unmagnetized quantum plasma with electrons with an arbitrary degeneracy degree. The equation of state for electrons follows from a local Fermi-Dirac distribution function and applies equally well both to fully degenerate and classical, nondegenerate limits. Ions are assumed to be cold. Quantum diffraction effects through the Bohm potential are also taken into account. A general coupling parameter valid for dilute and dense plasmas is proposed. The linear dispersion relation of the ion-acoustic waves is obtained and the ion-acoustic speed is discussed for the limiting cases of extremely dense or dilute systems. In the long-wavelength limit, the results agree with quantum kinetic theory. Using the reductive perturbation method, the appropriate Korteweg-de Vries equation for weakly nonlinear solutions is obtained and the corresponding soliton propagation is analyzed. It is found that soliton hump and dip structures are formed depending on the value of the quantum parameter for the degenerate electrons, which affect the phase velocities in the dispersive medium.
\end{abstract}

DOI: 10.1103/PhysRevE.92.053112

PACS number(s): 52.35.Fp, 52.35.Sb, 67.10.Db

\section{INTRODUCTION}

The study of degenerate plasma is important due to its applications, e.g., to strong laser produced plasmas [1], high density astrophysical plasmas such as in white dwarfs or neutron stars [2], or large density electronic devices (as in the drain region of $n^{+} n n^{+}$diodes [3]). In plasmas, the quantum effects are more relevant for electrons rather than ions because of their lower mass. The quantum nature of the charge carriers manifests with the inclusion of both the Pauli exclusion principle for fermions and the Heisenberg uncertainty principle due to the wavelike character of the particles. Accordingly, electrons obey the Fermi-Dirac statistics and their equation of state is determined using the Fermi-Dirac distribution. On the other hand, the quantum diffraction effects are usually modeled by means of quantum recoil terms in kinetic theory or the Bohm potential in fluid theory, besides higher-order gradient corrections [4,5].

Accordingly, the wave propagation in a degenerate plasma can be studied using at least two main approaches, i.e., kinetic and hydrodynamic models. In kinetic theory, the unperturbed electron distribution is frequently given by a Fermi-Dirac function, while in hydrodynamics, the momentum equation for electrons is made consistent with the equation of state of a degenerate electron Fermi gas [4,5]. In fluid models, the ionsound wave propagation in plasmas with degenerate electrons has been investigated by a number of authors [6-12], using the equation of state for a cold (fully degenerate) Fermi electron gas, with a negligible thermodynamic temperature. The energy distribution of a degenerate electron gas described by the Fermi-Dirac distribution is characterized by two independent parameters, one of which is the chemical potential, while the other is the thermodynamic temperature. On the other hand, the energy spread for the classical ideal electron gas obeying Maxwell-Boltzmann distribution is uniquely determined by the thermodynamic temperature. The equation of state for the fully degenerate electron gas so reduces to a one-parameter problem, i.e., the chemical potential. Therefore, it is of interest to study the linear and especially nonlinear wave propagation in the intermediate regime, depending on the competition between the two parameters, i.e., chemical potential and thermal temperature [13], including quantum diffraction.

Our treatment is especially relevant to borderline systems with $T \approx T_{F}$, which are neither strongly degenerate nor sufficiently well described by classical statistics, where $T$ and $T_{F}$ are, respectively, the electron thermodynamic and Fermi temperatures. A striking example is provided by inertial confinement fusion plasmas [14], with particle densities ranging from $10^{30}$ to $10^{32} \mathrm{~m}^{-3}$ and thermodynamic temperatures above $10^{7} \mathrm{~K}$. During laser irradiation of the solid target, quantum statistical effects tend to be more relevant immediately after compression, before the heating phase. Moreover, laboratory simulation of astrophysical scenarios involving dense plasmas better fit the intermediate quantum-classical regime [15]. For these reasons and potential applications on, e.g., ultrasmall semiconductor devices operating in a mixed dense-dilute regime [3], it is desirable to have a general macroscopic model covering both classical and quantum statistics, besides quantum diffraction.

Previously, Maafa [16] studied the ion-acoustic and Langmuir waves in a plasma with arbitrary degeneracy of electrons using classical kinetic theory, linearizing the Vlasov-Poisson system around a Fermi-Dirac equilibrium. Using quantum kinetic theory, Melrose and Mushtaq derived the electron-ion plasma low-frequency longitudinal response including quantum recoil, first for dilute (Maxwell-Boltzmann equilibrium) plasmas [17] and then for general degeneracy [18], in a Fermi-Dirac equilibrium. These works were restricted to linear waves only. Eliasson and Shukla [19] derived nonlinear quantum electron fluid equations by taking the moments of the 
evolution equation for the Wigner function in terms of a local Fermi-Dirac equilibrium with an arbitrary thermodynamic temperature. In this model, quantum diffraction manifests in terms of the Bohm potential. The high (classical) as well as the low (degenerate) temperature limits of the obtained fluid equations were also discussed in connection to Langmuir waves. Recently, Dubinov et al. [20] investigated the nonlinear theory of ion-acoustic waves in isothermal plasmas with arbitrary degeneracy, but without including quantum recoil. They presented the equation of state for ions and electrons by considering them as warm $(T \neq 0)$ Fermi gases. The nonlinear analysis was done using a Bernoulli pseudopotential approach. The ranges of the phase velocities of the periodic ion-acoustic waves and the soliton velocity were investigated. However, for simplicity, they ignored the quantum Bohm potential, which increases the order of the resulting dynamical equations. Our central issue here is to analyze the combined quantum statistical and quantum diffraction effects on linear and nonlinear ion-acoustic structures in plasmas, in an analytically simple (but hopefully not simplistic) approach.

The paper is organized in the following way. In Sec. II, the basic set of hydrodynamic equations is proposed and the barotropic equation of state is defined, for a general Fermi-Dirac equilibrium. In Sec. III, the linear dispersion relation for quantum ion-acoustic waves is derived, following the fluid model. Comparison with known results from quantum kinetic theory allows one to determine a fitting parameter in the quantum force, so that the macroscopic and microscopic approaches coincide in the long-wavelength limit. In Sec. IV, nonlinear wave structures are studied by means of the reductive perturbation method and the associated Korteweg-de Vries $(\mathrm{KdV})$ equation. The associated quantum soliton solution is obtained. Section V studies the possibility of forward and backward propagating solitons in real systems. Finally, Sec. VI presents some conclusions.

\section{MODEL EQUATIONS}

In order to study ion-acoustic waves in unmagnetized electron-ion plasmas with arbitrary electron temperature, we use the set of dynamic equations described as follows [4].

The ion continuity and momentum equations are, respectively, given by

$$
\begin{gathered}
\frac{\partial n_{i}}{\partial t}+\frac{\partial}{\partial x}\left(n_{i} u_{i}\right)=0, \\
\frac{\partial u_{i}}{\partial t}+u_{i} \frac{\partial u_{i}}{\partial x}=-\frac{e}{m_{i}} \frac{\partial \phi}{\partial x} .
\end{gathered}
$$

The momentum equation for the inertialess quantum electron fluid is given by

$$
0=e \frac{\partial \phi}{\partial x}-\frac{1}{n_{e}} \frac{\partial p}{\partial x}+\frac{\alpha \hbar^{2}}{6 m_{e}} \frac{\partial}{\partial x}\left(\frac{1}{\sqrt{n_{e}}} \frac{\partial^{2}}{\partial x^{2}} \sqrt{n_{e}}\right) .
$$

The Poisson equation is written as

$$
\frac{\partial^{2} \phi}{\partial x^{2}}=\frac{e}{\varepsilon_{0}}\left(n_{e}-n_{i}\right)
$$

where $\phi$ is the electrostatic potential. The ion fluid density and velocity are represented by $n_{i}$ and $u_{i}$, respectively, while $n_{e}$ is the electron fluid density. Also, $m_{e}$ and $m_{i}$ are the electron and ion masses, $-e$ is the electronic charge, $\varepsilon_{0}$ is the vacuum permittivity, and $\hbar$ is the reduced Planck constant. In Eq. (3), $\alpha$ is a dimensionless constant factor to be determined and $p=p\left(n_{e}\right)$ is the electron's fluid scalar pressure, to be specified by a barotropic equation of state obtained in the continuation.

The last term proportional to $\hbar^{2}$ on the right-hand side of the momentum equation for electrons is the quantum force, which arises due to the quantum Bohm potential, responsible for quantum diffraction or quantum tunneling effects due to the wavelike nature of the electrons. The dimensionless quantity $\alpha$ will be selected in order to exactly fit the kinetic theory linear dispersion relation in a three-dimensional FermiDirac equilibrium, as detailed in Sec. III. It is known that the qualitative role of the Bohm potential is to provide extra dispersion. However, the precise numerical coefficient in its definition is a debatable subject involving, e.g., the dimensionality and the temperature [21]. For instance, for a local Maxwell-Boltzmann equilibrium, Gardner [22] has found a factor $\alpha=1$. Frequently, the factor $\alpha$ is set in order to fit numerical results from kinetic theory [23], which is in the spirit of the present work. On the other hand, quantum effects on ions are ignored in the model in view of their large mass. For simplicity, ion temperature effects are also disregarded.

In order to derive the equation of state, consider a local quasiequilibrium Fermi-Dirac particle distribution function $f=f(\mathbf{v}, \mathbf{r}, t)$ for electrons [24], given by

$$
f(\mathbf{v}, \mathbf{r}, t)=\frac{A}{1+e^{\beta(\varepsilon-\mu)}},
$$

where $\beta=1 /\left(\kappa_{B} T\right), \varepsilon=m_{e} v^{2} / 2, v=|\mathbf{v}|$, and $\mu$ is the chemical potential regarded as a function of position $\mathbf{r}$ and time $t$. Besides, $\kappa_{B}$ is the Boltzmann constant, $T$ is the (constant) thermodynamic electron's temperature, and $\mathbf{v}$ is the velocity. In addition, $A$ is chosen to ensure the normalization $\int f d^{3} v=n_{e}$, so that

$$
A=-\frac{n_{e}}{\operatorname{Li}_{3 / 2}\left(-e^{\beta \mu}\right)}\left(\frac{\beta m_{e}}{2 \pi}\right)^{3 / 2}=2\left(\frac{m_{e}}{2 \pi \hbar}\right)^{3},
$$

the last equality following from the Pauli principle (the factor two is due to the electron's spin). Therefore, in the fluid description, $\mu$ and $A$ are supposed to be slowly varying functions of space and time. Equation (6) contains the polylogarithmic function $\operatorname{Li}_{v}(\eta)$ of index $v$, which can be generically defined [25] by

$$
\operatorname{Li}_{v}(\eta)=\frac{1}{\Gamma(\nu)} \int_{0}^{\infty} \frac{s^{\nu-1}}{e^{s} / \eta-1} d s,
$$

where $\Gamma(v)$ is the gamma function. We also observe that a three-dimensional equilibrium is assumed, although for electrostatic wave propagation, only one spatial variable $x$ is needed in the model equations.

The scalar pressure follows from the standard definition for an equilibrium with zero drift velocity,

$$
p=\frac{m_{e}}{3} \int f v^{2} d^{3} v
$$


yielding

$$
p=\frac{n_{e}}{\beta} \frac{\operatorname{Li}_{5 / 2}\left(-e^{\beta \mu}\right)}{\operatorname{Li}_{3 / 2}\left(-e^{\beta \mu}\right)} .
$$

It is worthwhile to consider some limiting cases of the barotropic equation of state. From Eq. (9), in the dilute plasma limit case with a local fugacity $e^{\beta \mu} \ll 1$ and using $\operatorname{Li}_{v}\left(-e^{\beta \mu}\right) \approx-e^{\beta \mu}$, one has

$$
p=n_{e} k_{B} T,
$$

which is the classical isothermal equation of state.

On the opposite, dense limit with a large local fugacity $e^{\beta \mu} \gg 1$, from $\operatorname{Li}_{v}\left(-e^{\beta \mu}\right) \approx-(\beta \mu)^{v} / \Gamma(\nu+1)$, the result is

$$
p=\frac{2}{5} n_{0} \varepsilon_{F}\left(\frac{n_{e}}{n_{0}}\right)^{5 / 3},
$$

which is the equation of state for a three-dimensional completely degenerate Fermi gas, expressed in terms of the equilibrium number density $n_{0}$. In Eq. (11), the electron's Fermi energy is $\varepsilon_{F}=\kappa_{B} T_{F}=\left[\hbar^{2} /\left(2 m_{e}\right)\right]\left(3 \pi^{2} n_{0}\right)^{2 / 3}$, which is the same as the equilibrium chemical potential in the fully degenerate case. In addition, $n_{0}$ is the equilibrium electron (and ion) number density.

The present treatment has similarities, as well as some different choices, in comparison to Eliasson and Shukla's work [19]. In this article, a local quasiequilibrium Fermi-Dirac distribution function was also employed. However, presently a nonconstant chemical potential is admitted. In addition, in Ref. [19], the focus was on situations involving onedimensional laser-plasma compression experiments, while here a three-dimensional isotropic equilibrium is assumed. Finally, the present work deals with low-frequency (ionacoustic) instead of high-frequency (Langmuir) waves.

In passing, from Eq. (6) one deduces the useful relation

$$
n_{e}=n_{0} \frac{\mathrm{Li}_{3 / 2}\left(-e^{\beta \mu}\right)}{\mathrm{Li}_{3 / 2}\left(-e^{\beta \mu_{0}}\right)},
$$

where $\mu_{0}$ is the equilibrium chemical potential, satisfying

$$
-\frac{n_{0}}{\mathrm{Li}_{3 / 2}\left(-e^{\beta \mu_{0}}\right)}\left(\frac{\beta m_{e}}{2 \pi}\right)^{3 / 2}=2\left(\frac{m_{e}}{2 \pi \hbar}\right)^{3}
$$

Using the equation of state (9), the chain rule, and the property $d \operatorname{Li}_{v}(\eta) / d \eta=(1 / \eta) \operatorname{Li}_{v-1}(\eta)$, the momentum equation (3) for the inertialess electron fluid becomes

$$
\begin{aligned}
0= & e \frac{\partial \phi}{\partial x}-\frac{1}{\beta n_{e}} \frac{\mathrm{Li}_{3 / 2}\left(-e^{\beta \mu}\right)}{\mathrm{Li}_{1 / 2}\left(-e^{\beta \mu}\right)} \frac{\partial n_{e}}{\partial x} \\
& +\frac{\alpha \hbar^{2}}{6 m_{e}} \frac{\partial}{\partial x}\left(\frac{1}{\sqrt{n_{e}}} \frac{\partial^{2}}{\partial x^{2}} \sqrt{n_{e}}\right) .
\end{aligned}
$$

Finally, using Eq. (12), we have the alternative form

$$
\begin{aligned}
0= & e \frac{\partial \phi}{\partial x}-\frac{1}{\beta n_{0}} \frac{\mathrm{Li}_{3 / 2}\left(-e^{\beta \mu_{0}}\right)}{\mathrm{Li}_{1 / 2}\left(-e^{\beta \mu}\right)} \frac{\partial n_{e}}{\partial x} \\
& +\frac{\alpha \hbar^{2}}{6 m_{e}} \frac{\partial}{\partial x}\left(\frac{1}{\sqrt{n_{e}}} \frac{\partial^{2}}{\partial x^{2}} \sqrt{n_{e}}\right),
\end{aligned}
$$

containing the minimal number of polylogarithmic functions with a nonconstant argument.

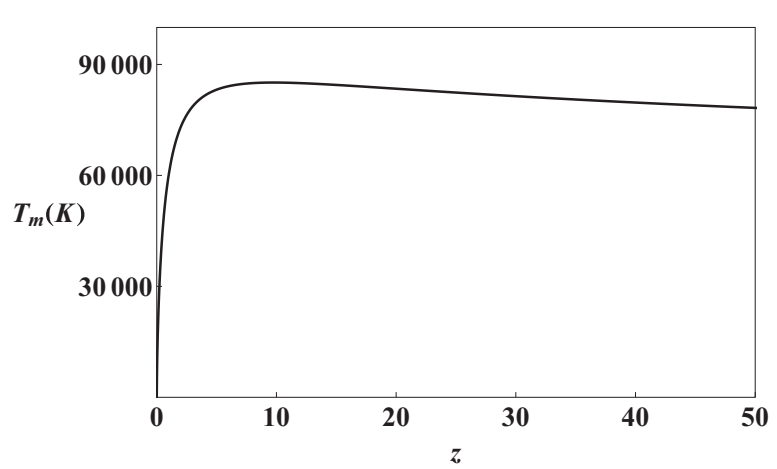

FIG. 1. Temperatures for the coupling parameter $g<1$ in Eq. (16) are above the curve, as a function of the fugacity $z=e^{\beta \mu_{0}}$.

It is worth noticing that the model does not include collisional damping, which is reasonable if the average electrostatic potential per particle $\langle U\rangle$ is much smaller than the corresponding average kinetic energy $\langle K\rangle$. For any degree of degeneracy, one can estimate $\langle U\rangle \approx e^{2} /\left(4 \pi \varepsilon_{0} r_{S}\right)$, where the Wigner-Seitz ratio $r_{S}$ is defined by $\left(4 \pi r_{S}^{3} / 3\right) n_{0}=1$. On the other hand, from $\langle K\rangle=\left[m_{e} /\left(2 n_{e}\right)\right] \int f v^{2} d^{3} v$ and evaluating on equilibrium, one derives the general coupling parameter,

$$
\begin{aligned}
g & \equiv \frac{\langle U\rangle}{\langle K\rangle}=\frac{1}{6}\left(\frac{4}{3 \pi^{2}}\right)^{1 / 3} \frac{e^{2} n_{0}^{1 / 3} \beta}{\varepsilon_{0}} \frac{\mathrm{Li}_{3 / 2}\left(-e^{\beta \mu_{0}}\right)}{\mathrm{Li}_{5 / 2}\left(-e^{\beta \mu_{0}}\right)} \\
& =-\frac{\sqrt{\beta m_{e} / 2}}{3^{4 / 3} \pi^{7 / 6}} \frac{e^{2}}{\varepsilon_{0} \hbar} \frac{\left[\mathrm{Li}_{3 / 2}^{2}\left(-e^{\beta \mu_{0}}\right)\right]^{2 / 3}}{\mathrm{Li}_{5 / 2}\left(-e^{\beta \mu_{0}}\right)}
\end{aligned}
$$

covering both degenerate and nondegenerate systems, in the nonrelativistic regime. In the last equality in Eq. (16), the expression (13) of the equilibrium density in terms of the equilibrium fugacity $z=e^{\beta \mu_{0}}$ and the temperature $T$ was used. In the dilute case, it follows from the properties of the polylogarithmic function that $g \propto\langle U\rangle /\left(\kappa_{B} T\right)$, while in the dense case $g \propto\langle U\rangle / \varepsilon_{F}$, with $\mu_{0} \approx \varepsilon_{F}$.

For both dilute or dense plasmas, the condition for low collisionality is that the interaction energy should be small in comparison to the kinetic energy, or $g \ll 1$ [26]. Using Eq. (16), the minimal temperature $T_{m}$ for low collisionality $(g<1$, relaxing the inequality sign) for both dilute and dense regimes follows from

$$
\kappa_{B} T>\kappa_{B} T_{m} \equiv \frac{m_{e}}{2 \times 3^{8 / 3} \pi^{7 / 3}}\left(\frac{e^{2}}{\varepsilon_{0} \hbar}\right)^{2}\left[\frac{\mathrm{Li}_{3 / 2}^{4 / 3}\left(-e^{\beta \mu_{0}}\right)}{\mathrm{Li}_{5 / 2}\left(-e^{\beta \mu_{0}}\right)}\right]^{2} .
$$

The result is shown in Fig. 1, where $T>T_{m}$ is equivalent to $g<1$. Starting from $z \approx 0$ and increasing the density, larger temperatures are needed for ideality, until reaching $z=$ $9.8, T=8.5 \times 10^{4} \mathrm{~K}$, corresponding to $n_{0}=2.9 \times 10^{29} \mathrm{~m}^{-3}$. For $z>9.8$, smaller temperatures are admitted, due to the Pauli blocking effect inhibiting collisions.

For the sake of comparison, instead of

$$
\langle K\rangle=\frac{3}{2} \kappa_{B} T \frac{\operatorname{Li}_{5 / 2}\left(-e^{\beta \mu_{0}}\right)}{\operatorname{Li}_{3 / 2}\left(-e^{\beta \mu_{0}}\right)},
$$




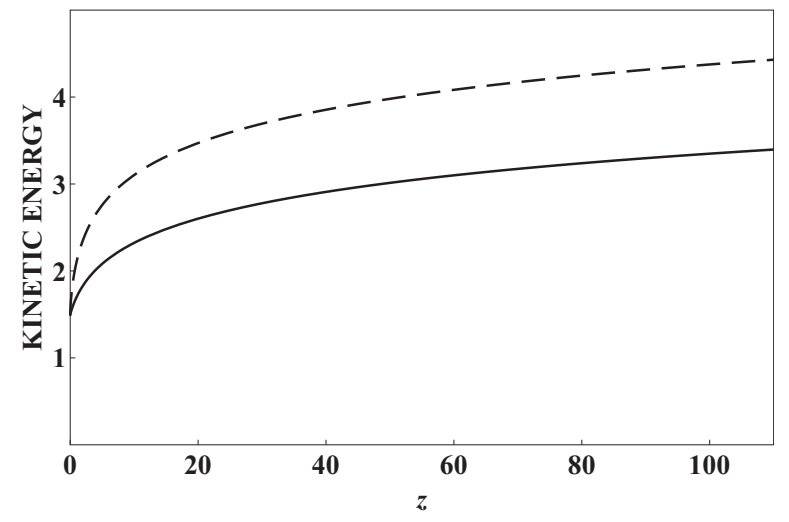

FIG. 2. Comparison between the average kinetic energy $\langle K\rangle-$ the continuous curve - given by Eq. (18) and the simpler form $\langle K\rangle_{z}$ the dashed curve-given by Eq. (19), as a function of the fugacity $z=e^{\beta \mu_{0}}$. Both energies are normalized to $\kappa_{B} T$.

Zamanian et al. used [27] the useful simpler expression

$$
\langle K\rangle_{Z}=\frac{3}{2} \kappa_{B} T+\frac{3}{5} \varepsilon_{F}
$$

as a measure of the kinetic energy per particle. More precisely, Ref. [27] employed the arithmetic sum of the thermal and Fermi energies, but in Eq. (19) we set some numerical factors to have agreement with the exact form in the dilute and ultradense cases where one has, respectively, $\langle K\rangle \approx 3 \kappa_{B} T / 2$ and $\langle K\rangle \approx$ $3 \varepsilon_{F} / 5$. In fact, using Eq. (13) expressing the density in terms of the fugacity and the temperature, as well as the expression of the Fermi energy, one finds

$$
\frac{\langle K\rangle_{Z}}{\kappa_{B} T}=\frac{3}{2}+\frac{3^{5 / 3}}{10}\left(\frac{\pi}{2}\right)^{1 / 3}\left[-\mathrm{Li}_{3 / 2}\left(-e^{\beta \mu_{0}}\right)\right]^{2 / 3},
$$

where the right-hand sides are functions of the fugacity only. This expression is shown in Fig. 2, compared to the more exact result found from Eq. (18). It is seen that the approximate form overestimates the kinetic energy, due to slow convergence. Nevertheless, by construction, for extreme degeneracy both quantities give the same numbers.

In the same spirit, one can define a general electron thermal velocity (in the sense of spreading of velocities) as $v_{T} \equiv \sqrt{2\langle K\rangle / m_{e}}$, which is found from Eq. (18),

$$
v_{T}=\left[\frac{3}{\beta m_{e}} \frac{\operatorname{Li}_{5 / 2}\left(-e^{\beta \mu_{0}}\right)}{\operatorname{Li}_{3 / 2}\left(-e^{\beta \mu_{0}}\right)}\right]^{1 / 2} .
$$

In the dilute case, one has $v_{T} \approx \sqrt{3 \kappa_{B} T / m_{e}}$, while in the dense case, $v_{T} \approx \sqrt{(6 / 5) \varepsilon_{F} / m_{e}}$.

For nondegenerate ions in strongly coupled plasma, the ion crystallization effects $[28,29]$ that appear due to viscoelasticity of the ion fluid in the ion momentum equation and cause damping of the ion-acoustic wave are ignored under the assumptions (in the three-dimensional version) $\tau_{m} \ll \omega_{p i}$ and $\partial \mathbf{u}_{i} / \partial t \gg$ $\left(\eta / \rho_{i}\right) \nabla^{2} \mathbf{u}_{i}+\left(1 / \rho_{i}\right)(\zeta+\eta / 3) \nabla\left(\nabla \cdot \mathbf{u}_{i}\right)$, where $\rho_{i}=n_{i} m_{i}$ is the ion mass density, $\tau_{m}$ is the viscoelastic relaxation time or memory function for ions, $\eta$ is the shear, and $\zeta$ are the bulk ion viscosity coefficients, respectively.

\section{LINEAR WAVES}

\section{A. Fluid theory}

We linearize the system given by Eqs. (1), (2), (4), and (15) by considering the first-order perturbations (with a subscript 1) relative to the equilibrium, as follows:

$$
\begin{aligned}
n_{i} & =n_{0}+n_{i 1}, n_{e}=n_{0}+n_{e 1}, \quad u_{i}=u_{i 1}, \\
\phi & =\phi_{1}, \quad \mu=\mu_{0}+\mu_{1} .
\end{aligned}
$$

The dispersion relation is obtained assuming plane wave excitations $\sim \exp [i(k x-\omega t)]$, yielding

$$
\omega^{2}=\frac{\omega_{p i}^{2} c_{s}^{2} k^{2}\left(1+\frac{\alpha \hbar^{2} k^{2}}{12 m_{e} m_{i} c_{s}^{2}}\right)}{\omega_{p i}^{2}+\left(1+\frac{\alpha \hbar^{2} k^{2}}{12 m_{e} m_{i} c_{s}^{2}}\right) c_{s}^{2} k^{2}},
$$

where

$$
c_{s}=\sqrt{\frac{1}{m_{i}}\left(\frac{\partial p}{\partial n_{e}}\right)_{0}}=\sqrt{\frac{1}{\beta m_{i}} \frac{\mathrm{Li}_{3 / 2}\left(-e^{\beta \mu_{0}}\right)}{\mathrm{Li}_{1 / 2}\left(-e^{\beta \mu_{0}}\right)}}
$$

plays the role of a generalized ion-acoustic speed and $\omega_{p i}=$ $\sqrt{n_{0} e^{2} /\left(m_{i} \varepsilon_{0}\right)}$ is the ion plasma frequency.

In the long-wavelength limit $\alpha \hbar^{2} k^{4} /\left(12 m_{e} m_{i}\right) \ll c_{s}^{2} k^{2} \ll$ $\omega_{\text {pi }}^{2}$, it follows from Eq. (22) that $\omega^{2} \approx c_{s}^{2} k^{2}$. In the dilute case with a small fugacity $e^{\beta \mu_{0}} \ll 1$, the well-known classical result $c_{s} \approx c_{s c} \equiv \sqrt{\kappa_{B} T / m_{i}}$ is verified. In the opposite, extremely degenerate case where the fugacity $e^{\beta \mu_{0}} \gg 1$, one finds $c_{s} \approx \sqrt{(2 / 3) \varepsilon_{F} / m_{i}}$, which is the ion-acoustic velocity for a three-dimensional ultradense plasma [16]. Finally, the very short-wavelength limit of the dispersion relation gives ion oscillations such that $\omega=\omega_{p i}$, both in the classical or quantum situations. This happens because the ions are no longer shielded by electrons when the wavelength is comparable to or smaller than the electron shielding length. It is interesting to note that taking the square root of both sides of Eq. (22) is identical to Eq. (4.5) in Ref. [30] for the completely degenerate plasma case, i.e., for $\alpha=1 / 3$.

Using Eq. (23), the ion-acoustic speed $c_{s}$ normalized to the purely classical expression $c_{s c}$ against $z=e^{\beta \mu_{0}}$ is shown in Fig. 3. It can be seen that as the value of $z$ increases (i.e., the degeneracy of electrons and plasma density increase), the ion-acoustic speed also increases.

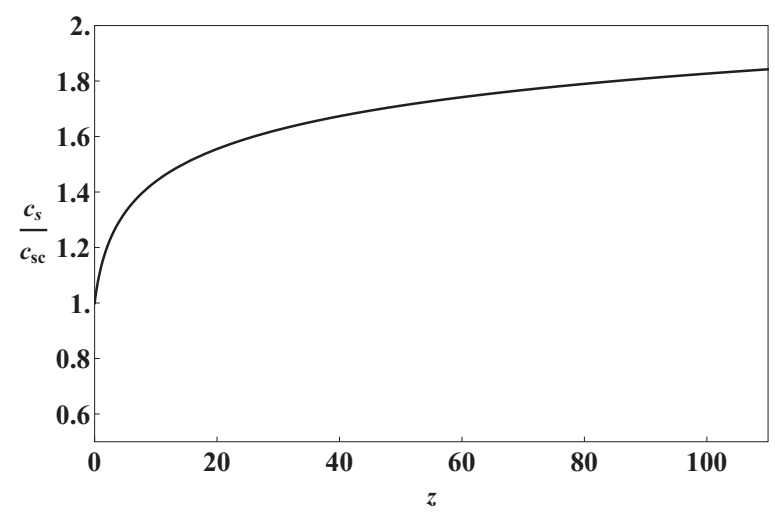

FIG. 3. The profile of the ion-acoustic speed $c_{s}$ from Eq. (23), normalized to the classical expression $c_{s c}$, as a function of the fugacity $z=e^{\beta \mu_{0}}$. 


\section{B. Kinetic theory}

To endorse the macroscopic modeling and to set the value of the parameter $\alpha$ in front of the quantum force, it is useful to compare with the microscopic (quantum kinetic) results. Considering the Wigner-Poisson system [4] involving a cold ionic species and electrons, it is straightforward to derive the linear dispersion relation,

$$
1=\frac{\omega_{p i}^{2}}{\omega^{2}}+\frac{\omega_{p e}^{2}}{n_{0}} \int \frac{f_{0}(\mathbf{v}) d^{3} v}{(\omega-\mathbf{k} \cdot \mathbf{v})^{2}-\hbar^{2} k^{4} /\left(4 m_{e}^{2}\right)},
$$

where $f_{0}(\mathbf{v})$ is the equilibrium electronic Wigner function and $\omega_{p e}=\sqrt{n_{0} e^{2} /\left(m_{e} \varepsilon_{0}\right)}$ is the electron plasma frequency.

The longitudinal response of an electron-ion plasma in a Fermi-Dirac equilibrium,

$$
f_{0}(\mathbf{v})=\frac{A}{1+e^{\beta\left(\varepsilon-\mu_{0}\right)}},
$$

has been calculated in [18], where $A$ and $\mu_{0}$ are obtained from Eq. (13). Including the first-order correction from quantum recoil, the result is

$$
\begin{aligned}
1= & \frac{\omega_{p i}^{2}}{\omega^{2}}-\frac{\omega_{p i}^{2}}{c_{s}^{2} k^{2}} \\
& \times\left\{1-\frac{m_{e}\left[\omega^{2}+\hbar^{2} k^{4} /\left(12 m_{e}^{2}\right)\right]}{k^{2} \kappa_{B} T} \frac{\mathrm{Li}_{-1 / 2}\left(-e^{\beta \mu_{0}}\right)}{\mathrm{Li}_{1 / 2}\left(-e^{\beta \mu_{0}}\right)}\right\},
\end{aligned}
$$

which follows from Eq. (29) of [18], in a different notation. The first and second terms in the right-hand side of Eq. (26) are, respectively, the ionic and electronic responses of the plasma.

For the treatment of low-frequency waves, for simplicity it is sufficient to consider the static electronic response, so that we set $\omega \approx 0$ in the last term of Eq. (26). From inspection, and since we want to retain the first-order quantum correction, this approximation requires $\omega^{2} \ll \hbar^{2} k^{4} /\left(12 m_{e}^{2}\right)$. Under the long-wavelength assumption $k^{2} c_{s}^{2} \ll \omega_{p i}^{2}$ and the leading order result $\omega^{2} \approx c_{s}^{2} k^{2}$, it follows that

$$
\frac{12 m_{e}^{2} c_{s}^{2}}{\hbar^{2}} \ll k^{2} \ll \frac{\omega_{p i}^{2}}{c_{s}^{2}} .
$$

Taking into account the ion-acoustic velocity from Eq. (23), from Eq. (27) one has the necessary condition

$$
\frac{\beta^{2} \hbar^{2} \omega_{p i}^{2}}{12} \gg\left(\frac{m_{e}}{m_{i}}\right)^{2}\left[\frac{\mathrm{Li}_{3 / 2}\left(-e^{\beta \mu_{0}}\right)}{\mathrm{Li}_{1 / 2}\left(-e^{\beta \mu_{0}}\right)}\right]^{2} .
$$

The combined low-frequency and long-wavelength requirement (28) is more easily worked out in the dilute $\left(e^{\beta \mu_{0}} \ll\right.$ $1)$ and fully degenerate $\left(e^{\beta \mu_{0}} \gg 1\right)$ cases. For hydrogen plasma and using the appropriate asymptotic expansions of the polylogarithmic functions, one finds $n_{0} / T^{2} \gg 3.5 \times$ $10^{16} \mathrm{~m}^{-3} \mathrm{~K}^{-2}$ in the nondegenerate situation, and $n_{0} \ll 4.5 \times$ $10^{37} \mathrm{~m}^{-3}$ for very dense systems. It is seen that nondegenerate plasmas satisfy (28) more easily in denser and colder plasmas, while fully degenerate plasmas safely fit the assumptions, except for extreme densities (e.g., neutron star), which would deserve a relativistic treatment. Otherwise, there would be the need to retain the full electronic response in Eq. (26). As a consequence, a somewhat more involved dispersion relation would be found. In fact, using $n_{0}$ from Eq. (13), it can be

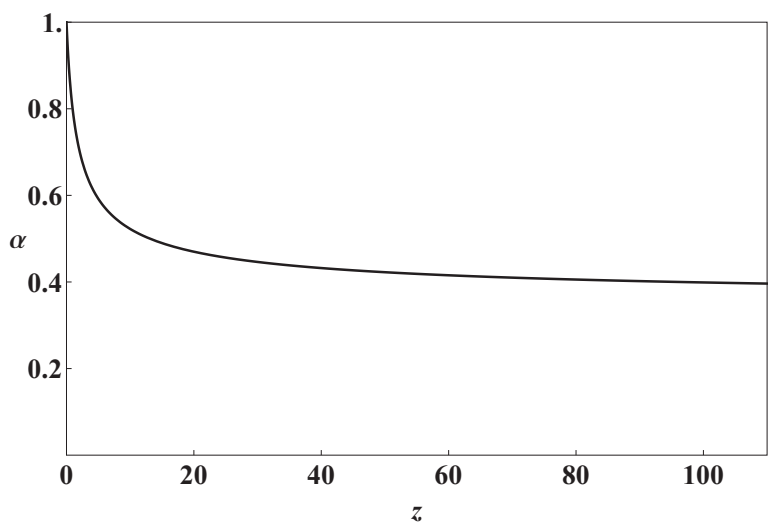

FIG. 4. Behavior of the numerical coefficient $\alpha$ in Eq. (33), as a function of the fugacity $z=\exp \left(\beta \mu_{0}\right)$.

shown that the necessary condition (28) is safely attended for all fugacities, as far as $T \ll 10^{9} \mathrm{~K}$, which is reasonable in view of the nonrelativistic assumption.

Dropping $\omega$ in the electronic response, Eq. (26) considerably simplifies, reducing to

$$
1=\frac{\omega_{p i}^{2}}{\omega^{2}}-\frac{\omega_{p i}^{2}}{c_{s}^{2} k^{2}}\left[1-\frac{\hbar^{2} k^{2}}{12 m_{e} \kappa_{B} T} \frac{\mathrm{Li}_{-1 / 2}\left(-e^{\beta \mu_{0}}\right)}{\operatorname{Li}_{1 / 2}\left(-e^{\beta \mu_{0}}\right)}\right] .
$$

Solving for the frequency yield,

$$
\omega^{2}=\frac{\omega_{p i}^{2} c_{s}^{2} k^{2}}{\omega_{p i}^{2}+\left[1-\frac{\beta^{2} \hbar^{2} \omega_{p e}^{2}}{12} \frac{\mathrm{Li}_{-1 / 2}\left(-e^{\beta \mu_{0}}\right)}{\mathrm{Li}_{3 / 2}\left(-e^{\beta \mu_{0}}\right)}\right] c_{s}^{2} k^{2}} .
$$

The expression from kinetic theory is valid for wavelengths larger than the electron shielding length of the system. To make a comparison with the result from fluid theory, it is necessary to expand (30) for small wave numbers,

$$
\begin{aligned}
\omega^{2}= & c_{s}^{2} k^{2}\left\{1+\left[-1+\frac{\beta^{2} \hbar^{2} \omega_{p e}^{2}}{12} \frac{\mathrm{Li}_{-1 / 2}\left(-e^{\beta \mu_{0}}\right)}{\mathrm{Li}_{3 / 2}\left(-e^{\beta \mu_{0}}\right)}\right] \frac{c_{s}^{2} k^{2}}{\omega_{p i}^{2}}\right\} \\
& +O\left(k^{6}\right) .
\end{aligned}
$$

Next, expand the fluid theory expression (22) for small wave numbers,

$$
\omega^{2}=c_{s}^{2} k^{2}\left[1+\left(-1+\frac{\alpha \hbar^{2} \omega_{p i}^{2}}{12 m_{e} m_{i} c_{s}^{4}}\right) \frac{c_{s}^{2} k^{2}}{\omega_{p i}^{2}}\right]+O\left(k^{6}\right) .
$$

Equations (31) and (32) are equivalent provided we set

$$
\alpha=\frac{\operatorname{Li}_{3 / 2}\left(-e^{\beta \mu_{0}}\right) \mathrm{Li}_{-1 / 2}\left(-e^{\beta \mu_{0}}\right)}{\left[\mathrm{Li}_{1 / 2}\left(-e^{\beta \mu_{0}}\right)\right]^{2}},
$$

which is our ultimate choice. Therefore, to comply with the results of kinetic theory on quantum ion-acoustic waves in a three-dimensional Fermi-Dirac equilibrium, the numerical coefficient in the quantum force has to be a function of the fugacity. In particular, with $z=\exp \left(\beta \mu_{0}\right)$, we have $\alpha \rightarrow 1$ for $z \rightarrow 0$ and $\alpha \rightarrow 1 / 3$ as $z \rightarrow \infty$. Moreover, as seen in Fig. 4, the coefficient $\alpha$ is a monotonically decreasing function of the fugacity, showing that the quantum force becomes less effective in denser systems. The result $\alpha \rightarrow 1$ for nondegenerate systems agrees with the quantum hydrodynamic model 
for semiconductor devices derived in [22], while $\alpha \rightarrow 1 / 3$ agrees with $[31,32]$ in the fully degenerate case. On the other hand, high-frequency waves such as quantum Langmuir waves would be correctly described by a value $\alpha=3$, in order to reproduce the Bohm-Pines [33] dispersion relation $\omega^{2}=$ $\omega_{p e}^{2}+(3 / 5) k^{2} v_{F}^{2}+(1 / 4) \hbar^{2} k^{4} / m_{e}^{2}$, where $v_{F}=\left(2 E_{F} / m_{e}\right)^{1 / 2}$ is the Fermi velocity.

The detailed account of the collisionless damping of quantum ion-acoustic waves has been considered in [18], where the damping rate is shown to be small, as long as the ion temperature is much smaller than the electron temperature of the plasma.

\section{NONLINEAR WAVES}

Having performed the analysis of linear quantum ionacoustic waves, it is worthwhile to consider the nonlinear structures which are accessible through our hydrodynamic model.

From now on, it is useful to define the rescaling

$$
\begin{aligned}
\tilde{x} & =\frac{\omega_{p i} x}{c_{s}}, \tilde{t}=\omega_{p i} t, \quad \tilde{n}_{e, i}=\frac{n_{e, i}}{n_{0}}, \\
\tilde{u}_{i} & =\frac{u_{i}}{c_{s}}, \Phi=\frac{e \phi}{m_{i} c_{s}^{2}},
\end{aligned}
$$

so that the model equations (1)-(4) can be written in a normalized form as follows:

$$
\begin{gathered}
\frac{\partial \tilde{n}_{i}}{\partial \tilde{t}}+\frac{\partial}{\partial \tilde{x}}\left(\tilde{n}_{i} \tilde{u}_{i}\right)=0 \\
\frac{\partial \tilde{u}_{i}}{\partial \tilde{t}}+\tilde{u}_{i} \frac{\partial}{\partial \tilde{x}} \tilde{u}_{i}=-\frac{\partial \Phi}{\partial \tilde{x}} \\
0=\frac{\partial \Phi}{\partial \tilde{x}}-\frac{\operatorname{Li}_{1 / 2}\left(-e^{\beta \mu_{0}}\right)}{\operatorname{Li}_{1 / 2}\left(-e^{\beta \mu}\right)} \frac{\partial \tilde{n}_{e}}{\partial \tilde{x}}+\frac{H^{2}}{2} \frac{\partial}{\partial \tilde{x}}\left(\frac{1}{\sqrt{\tilde{n}_{e}}} \frac{\partial^{2}}{\partial \tilde{x}^{2}} \sqrt{\tilde{n}_{e}}\right), \\
\frac{\partial^{2} \Phi}{\partial \tilde{x}^{2}}=\tilde{n}_{e}-\tilde{n}_{i}
\end{gathered}
$$

introducing the quantum parameter $H$ given by

$$
H=\frac{\beta \hbar \omega_{p e}}{\sqrt{3}}\left[\frac{\mathrm{Li}_{-1 / 2}\left(-e^{\beta \mu_{0}}\right)}{\mathrm{Li}_{3 / 2}\left(-e^{\beta \mu_{0}}\right)}\right]^{1 / 2} .
$$

In the dilute or fully degenerate cases, one has, respectively, $H \approx \beta \hbar \omega_{p e} / \sqrt{3}$ or $H \approx \hbar \omega_{p e} /\left(2 \varepsilon_{F}\right)$. Moreover, from Eq. (12),

$$
\tilde{n}_{e}=\frac{\mathrm{Li}_{3 / 2}\left(-e^{\beta \mu}\right)}{\operatorname{Li}_{3 / 2}\left(-e^{\beta \mu_{0}}\right)} .
$$

In the following, for simplicity, the tilde will be omitted in the normalized quantities.

In order to find a nonlinear evolution equation, a stretching of the independent variables $x, t$ is defined as follows $[6,12]$ :

$$
\xi=\varepsilon^{1 / 2}\left(x-V_{0} t\right), \quad \tau=\varepsilon^{3 / 2} t,
$$

where $\varepsilon$ is a small parameter and $V_{0}$ is the phase velocity of the wave to be determined later. The perturbed quantities can be expanded in powers of $\varepsilon$,

$$
\begin{aligned}
n_{i} & =1+\varepsilon n_{i 1}+\varepsilon^{2} n_{i 2}+\cdots, \\
n_{e} & =1+\varepsilon n_{e 1}+\varepsilon^{2} n_{e 2}+\cdots, \\
u_{i} & =\varepsilon u_{i 1}+\varepsilon^{2} u_{i 2}+\cdots, \\
\Phi & =\varepsilon \Phi_{1}+\varepsilon^{2} \Phi_{2}+\cdots, \\
\mu & =\mu_{0}+\varepsilon \mu_{1}+\varepsilon^{2} \mu_{2}+\cdots .
\end{aligned}
$$

The lowest order equations give

$$
n_{i 1}=u_{i 1}=n_{e 1}=\Phi_{1},
$$

and

$$
V_{0}= \pm 1
$$

which is the normalized phase velocity of the ion-acoustic wave in plasmas with arbitrary degeneracy of electrons. From now on, we set $V_{0}=1$ without loss of generality.

Now collecting the next higher-order terms, we have

$$
\begin{gathered}
\frac{\partial n_{i 2}}{\partial \xi}=\frac{\partial n_{i 1}}{\partial \tau}+\frac{\partial}{\partial \xi}\left(n_{i 1} u_{i 1}\right) \\
+\frac{\partial u_{i 1}}{\partial \tau}+u_{i 1} \frac{\partial u_{i 1}}{\partial \xi}+\frac{\partial \Phi_{2}}{\partial \xi}, \\
\frac{\partial n_{e 2}}{\partial \xi}=\frac{\partial \Phi_{2}}{\partial \xi}+\alpha n_{e 1} \frac{\partial n_{e 1}}{\partial \xi}+\frac{H^{2}}{4} \frac{\partial^{3} n_{e 1}}{\partial \xi^{3}} .
\end{gathered}
$$

Using the next higher-order Poisson's equation together with Eqs. (42), (44), and (45) yield the KdV equation for ion-acoustic waves in plasmas with arbitrary degeneracy of electrons,

$$
\frac{\partial \Phi_{1}}{\partial \tau}+a \Phi_{1} \frac{\partial \Phi_{1}}{\partial \xi}+b \frac{\partial^{3} \Phi_{1}}{\partial \xi^{3}}=0,
$$

where the nonlinear and dispersive coefficients $a$ and $b$ are, respectively, defined as

$$
a=\frac{3-\alpha}{2}, b=\frac{1}{2}\left(1-\frac{H^{2}}{4}\right) .
$$

In the fully classical limit (nondegeneracy and no Bohm potential), one has $a=1, b=1 / 2$, recovering the $\mathrm{KdV}$ equation for classical ion-acoustic waves [34]. The effect of arbitrary degeneracy of electrons appears in both the nonlinear and dispersive coefficients in the $\mathrm{KdV}$ equation (46).

It is easy to derive traveling wave solutions for the problem. One of them is the one-soliton solution of the $\mathrm{KdV}$ equation (46) given by

$$
\Phi_{1}=D \operatorname{sech}^{2}\left(\frac{\eta}{W}\right),
$$

where $D=3 u_{0} / a$ and $W=\sqrt{4 b / u_{0}}$ are, respectively, the height and width of the soliton. The polarity of the soliton depends on the sign of $D$. In the comoving frame, one has $\eta=\xi-u_{0} \tau$, where $u_{0}$ is the speed of the nonlinear structure. Decaying boundary conditions in the comoving system were used. For a given perturbation speed, one concludes that larger degeneracy (larger $a, b)$ gives a smaller scaled amplitude and a larger scaled width. This is because it becomes harder to accommodate more fermions in a localized wave packet 


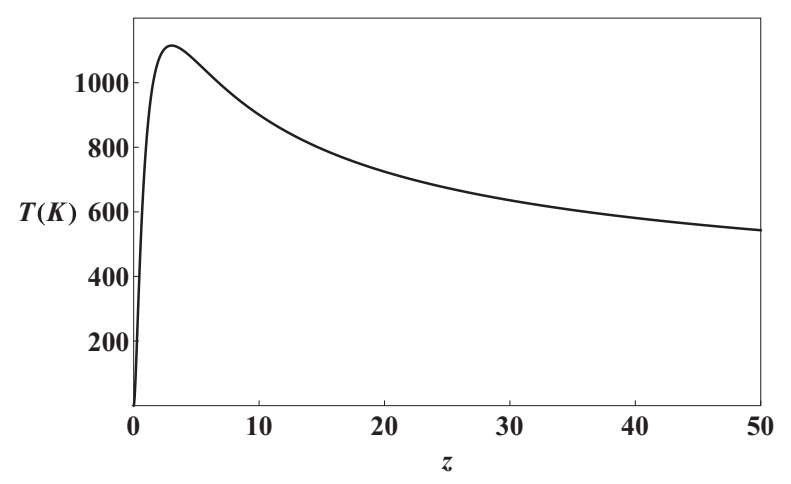

FIG. 5. The temperatures for $H>2$ satisfying Eq. (50) are below the curve, where $z=e^{\beta \mu_{0}}$.

under strong degeneracy. The transformed coordinate $\eta$ can be written as $\eta=\varepsilon^{1 / 2} \tilde{\eta}$, where $\tilde{\eta}=x-V t$ and $V=V_{0}+\varepsilon u_{0}$ is the soliton velocity in the laboratory frame.

It can be seen from the relation (47) that the dispersive coefficient $b$ disappears at $H=2$. In principle, the lack of a dispersive term eventually yields the formation of a shock. However, actually, in this case a dispersive contribution could be obtained from a higher-order perturbation theory, as occurs in the Kawahara equation [35]. In the present context of quantum ion-acoustic nonlinear waves, the soliton solution can exist only for $H \neq 2$, with a proper balance between dispersion and nonlinearity. Notice that for $H<2$, the soliton velocity is positive, i.e., $u_{0}>0$ (which means $V>V_{0}$ and it moves with supersonic speed) and we have a hump (bright) soliton structure since $a>0$ and $D>0$. However, for the $H>2$ case, the dispersive coefficient becomes negative, i.e., $b<0$, so that the soliton solution will exist only if $u_{0}<0$ (i.e., $V<V_{0}$ soliton moves with subsonic speed), since the width $W$ should have real values. As $u_{0}$ is negative in the $H>2$ case, the nonlinearity coefficient remains positive, i.e., $a>0$, and therefore $D<0$ which gives a dip (or dark) soliton instead of a hump (or bright) structure [36]. In brief, the model predicts hump solitons for the $H<2$ case and dip solitons for $H>2$. Finally, in the special fine tuning case with $H=2$, there is a shock instead of solitonic solutions, at least within the present order of perturbation theory.

\section{ON BRIGHT AND DARK PROPAGATING SOLITONS}

The qualitative differences of quantum ion-acoustic soliton propagation for $H<2$ or $H>2$ deserve a closer examination of the associated physical conditions. First, the quantum parameter in Eq. (39) can be reexpressed according to

$$
H^{2}=-\frac{1}{3 \pi}\left(\frac{m_{e}}{2 \pi \kappa_{B} T}\right)^{1 / 2} \frac{e^{2}}{\varepsilon_{0} \hbar} \mathrm{Li}_{-1 / 2}\left(-e^{\beta \mu_{0}}\right),
$$

where the equilibrium density in Eq. (13) was employed. From the last equation, one finds that $H>2$ occurs for sufficiently small temperatures, or

$$
\kappa_{B} T<\frac{m_{e}}{288 \pi^{3}}\left(\frac{e^{2}}{\varepsilon_{0} \hbar}\right)^{2}\left[\mathrm{Li}_{-1 / 2}\left(-e^{\beta \mu_{0}}\right)\right]^{2},
$$

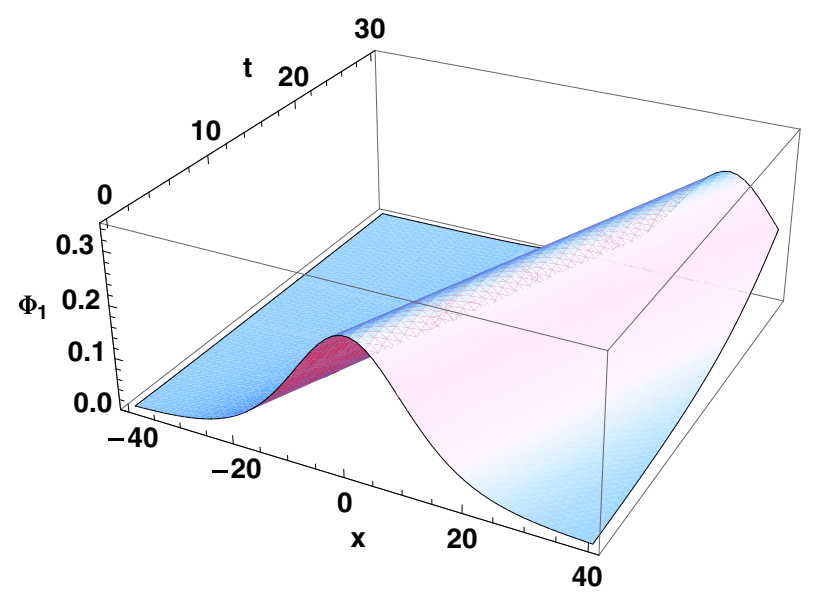

FIG. 6. (Color online) Hump soliton structure for $H<2$ is shown moving with supersonic speed in laboratory frame. The soliton hump corresponds to $T=10^{5} \mathrm{~K}, z=5, u_{0}=0.1, \varepsilon=0.1$ for which $H=0.64, n_{0}=3.5 \times 10^{29} \mathrm{~m}^{-3}, \omega_{p e}=3.3 \times 10^{16} \mathrm{~s}^{-1}$, respectively.

as illustrated in Fig. 5. Low-temperature plasmas with $T<10^{3} \mathrm{~K}$ are therefore candidates for the peculiar dark solitons. Starting from $z=e^{\beta \mu_{0}} \approx 0$, the maximal temperature increases until $z=3, T=1.1 \times 10^{3} \mathrm{~K}$ (corresponding to $n_{0}=$ $\left.3.0 \times 10^{26} \mathrm{~m}^{-3}\right)$, when it starts to decrease.

As an example, in Figs. 6 and 7, the two classes of quantum ion-acoustic bright or dark solitons are shown, following Eq. (48). The bright soliton $(H<2)$ moves with supersonic speed while the dark soliton $(H>2)$ moves with subsonic speed.

On the other hand, it is interesting to examine the conditions for weak coupling as deduced in the present theory. Combining the weak coupling condition yielding the minimal temperature in Eq. (17) with Eq. (49) gives an upper bound on the quantum diffraction parameter, or

$$
H^{2}<H_{M}^{2} \equiv-\left(\frac{3}{\pi}\right)^{1 / 3} \frac{\mathrm{Li}_{-1 / 2}\left(-e^{\beta \mu_{0}}\right) \mathrm{Li}_{5 / 2}\left(-e^{\beta \mu_{0}}\right)}{\left[\operatorname{Li}_{3 / 2}^{2}\left(-e^{\beta \mu_{0}}\right)\right]^{2 / 3}},
$$

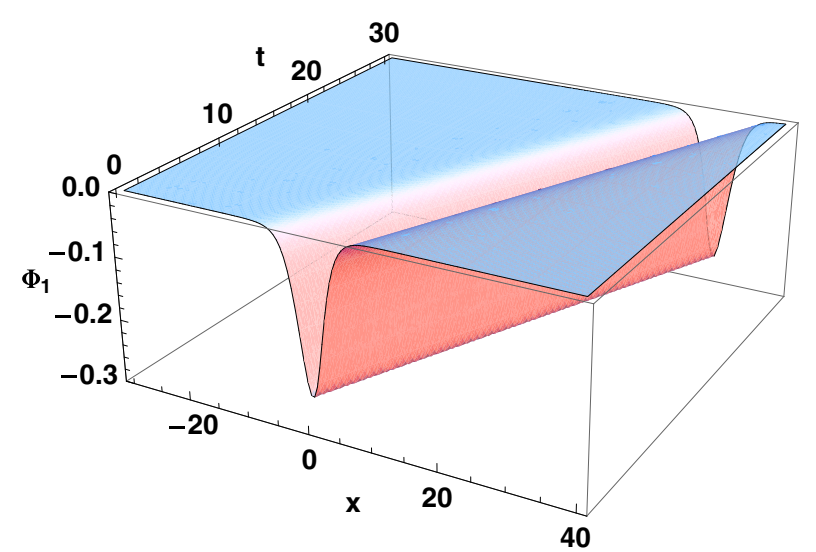

FIG. 7. (Color online) Dip soliton structure for $H>2$ is shown moving with subsonic speed in laboratory frame. The soliton dip is obtained at $T=10^{3} \mathrm{~K}, z=5, u_{0}=-0.1$, for which $H=2.03$, $n_{0}=3.5 \times 10^{26} \mathrm{~m}^{-3}, \omega_{p e}=1.1 \times 10^{15} \mathrm{~s}^{-1}$, respectively. 


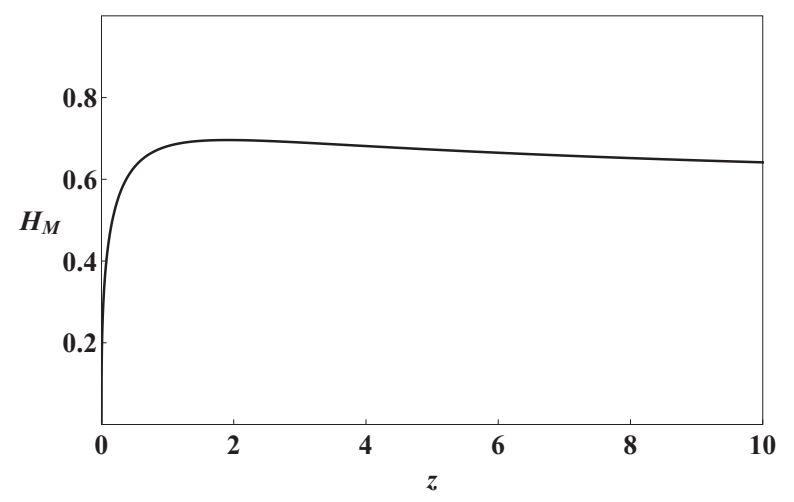

FIG. 8. Maximal quantum parameter $H_{M}$ satisfying the weak coupling assumption, according to Eq. (51), where $z=e^{\beta \mu_{0}}$.

which is shown in Fig. 8. It follows that large $H>2$ values fall within the strongly coupled regime where coupling parameter $g$ for degenerate electrons may become near or greater than one.

Nevertheless, considering ion-acoustic waves at least, a strong coupling between electrons will not be the main aspect of the dynamics. Although the complete analysis of the strongly coupled plasma regime is beyond the scope of this work, some conclusions can be found from the simplest way to introduce nonideality for electrons, namely, the addition of a dissipation term $-\omega_{\text {coll }} u_{e}$ in the right-hand side of Eq. (3), where $u_{e}$ is the electron fluid velocity and $\omega_{\text {coll }}$ is the electronelectron collision frequency. Using the continuity equation for electrons, it is possible to estimate $\omega n_{e 1} \approx k n_{0} u_{e 1}$, where $n_{e 1}$ and $u_{e 1}$ are the first-order perturbations of the electron fluid density and velocity. Finally, with $\omega \approx c_{s} k$, one finds that the dissipation term is negligible with respect to the pressure term provided $\omega \gg\left(m_{e} / m_{i}\right) \omega_{\text {coll }}$, which is always satisfied within the inertialess electrons assumption. We note that according to the Landau expression [37], one has in the nondegenerate case

$$
\frac{\omega_{\mathrm{coll}}}{\omega_{\mathrm{pe}}} \approx \frac{\ln \Lambda}{\Lambda},
$$

where $\Lambda \sim 1 / g^{3 / 2}$ is the plasma parameter. In the fully degenerate case, the right-hand side of Eq. (52) needs to be multiplied by the Pauli blocking factor $\kappa_{B} T / \varepsilon_{F}$. The conclusion is that except for very high $g \gg 1$, the electronelectron coupling can be neglected as long as the inertialess assumption is valid.

\section{CONCLUSION}

The linear and nonlinear ion-acoustic waves in a nonrelativistic quantum plasma with arbitrary degeneracy of electrons have been investigated. Besides degeneracy, the quantum diffraction effect of electrons was also included, in terms of the Bohm potential. The linear dispersion relation for quantum ion-acoustic waves was found in terms of a generalized ion-acoustic speed, valid for both the dilute and dense cases. The numerical factor $\alpha$ in front of the quantum force in the macroscopic model was fixed in order to comply with the kinetic theory results. The corresponding $\mathrm{KdV}$ equation was obtained using the reductive perturbation method. The possible classes of propagating solitons, namely, bright for $H<2$ moving with supersonic speed and dark for the $H>2$ case moving with subsonic speed, were discussed, where $H$ is a measure of the strength of quantum diffraction effects arising from the Bohm potential. To conclude, the derivation covers both the basic quantum effects in plasmas (arising, respectively, from quantum statistics and wavelike behavior of the charge carriers), in both the dilute and dense regimes. For instance, from Eq. (48), the scaled amplitude of the soliton becomes smaller for larger degeneracy, with $D=3 u_{0}$ for $\alpha=1$ (nondegenerate case) and $D=9 u_{0} / 4$ for $\alpha=1 / 3$ (fully degenerate case). The results are useful for the understanding of ion-acoustic wave propagation in an unmagnetized quantum plasma with arbitrary degeneracy of electrons.

\section{ACKNOWLEDGMENTS}

F.H. acknowledges CNPq (Conselho Nacional de Desenvolvimento Científico e Tecnológico) for financial support. S.M. acknowledges CNPq and TWAS (The World Academy of Sciences) for support through a CNPq-TWAS postdoctoral fellowship.
[1] S. H. Glenzer, O. L. Landen, P. Neumayer, R. W. Lee, K. Widmann, S. W. Pollaine, R. J. Wallace, G. Gregori, A. Höll, T. Bornath, R. Thiele, V. Schwarz, W.-D. Kraeft, and R. Redmer, Phys. Rev. Lett. 98, 065002 (2007).

[2] A. K. Harding and D. Lai, Rep. Prog. Phys. 69, 2631 (2006).

[3] A. Jüngel, Transport Equations for Semiconductors (Springer, Berlin, 2009).

[4] F. Haas, Quantum Plasmas: An Hydrodynamic Approach (Springer, New York, 2011).

[5] D. Melrose, Quantum Plasmadynamics - Unmagnetized Plasmas (Springer-Verlag, New York, 2008).

[6] F. Haas, L. G. Garcia, J. Goedert, and G. Manfredi, Phys. Plasmas 10, 3858 (2003).

[7] G. Manfredi and F. Haas, Phys. Rev. B 64, 075316 (2001).
[8] P. K. Shukla and S. Ali, Phys. Plasmas 12, 114502 (2005).

[9] R. Sabry, W. M. Moslem, and P. K. Shukla, Phys. Lett. A 372, 5691 (2008).

[10] U. M. Abdelsalam, W. M. Moslem, and P. K. Shukla, Phys. Plasmas 15, 052303 (2008).

[11] A. E. Dubinov and A. A. Dubinova, Plasma Phys. Rep. 33, 859 (2007).

[12] S. Mahmood and F. Haas, Phys. Plasmas 21, 102308 (2014).

[13] A. E. Dubinov and A. A. Dubinova, Plasma Phys. Rep. 34, 403 (2008).

[14] G. Manfredi and J. Hurst, Plasma Phys. Control. Fusion 57, 054004 (2015).

[15] J. E. Cross, B. Reville, and G. Gregori, Astrophys. J. 795, 59 (2014). 
[16] N. Maafa, Phys. Scr. 48, 351 (1993).

[17] A. Mushtaq and D. B. Melrose, Phys. Plasmas 16, 102110 (2009).

[18] D. B. Melrose and A. Mushtaq, Phys. Rev. E 82, 056402 (2010).

[19] B. Eliasson and P. K. Shukla, Phys. Scr. 78, 025503 (2008).

[20] A. E. Dubinov, A. A. Dubinova, and M. A. Sazokin, J. Commun. Tech. Elec. 55, 907 (2010).

[21] J. R. Barker and D. K. Ferry, Semicond. Sci. Technol. 13, A135 (1998).

[22] C. L. Gardner, SIAM J. Appl. Math. 54, 409 (1994).

[23] H. L. Rubin, T. R. Govindan, J. P. Kreskovski, and M. A. Stroscio, Solid State Electron. 36, 1697 (1993).

[24] R. K. Pathria and P. D. Beale, Statistical Mechanics, 3rd ed. (Elsevier, New York, 2011).

[25] L. Lewin, Polylogarithms and Associated Functions (North Holland, New York, 1981).

[26] A. I. Akhiezer, I. A. Akhiezer, R. V. Polovin, A. G. Sitenko, and K. N. Stepanov, Plasma Electrodynamics, Vol. I (Pergamon, Oxford, 1975).
[27] J. Zamanian, M. Marklund, and G. Brodin, New J. Phys 12, 043019 (2010)

[28] P. K. Shukla and B. Eliasson, Rev. Mod. Phys. 83, 885 (2011).

[29] A. P. Misra and P. K. Shukla, Phys. Rev. E 85, 026409 (2012).

[30] B. Eliasson and P. K. Shukla, J. Plasma Phys. 76, 7 (2010).

[31] D. Michta, F. Graziani, and M. Bonitz, Contrib. Plasma Phys. 55, 437 (2015).

[32] M. Akbari-Moghanjoughi, Phys. Plasmas 22, 022103 (2015).

[33] D. Bohm and D. Pines, Phys. Rev. 92, 609 (1953).

[34] R. C. Davidson, Methods in Nonlinear Plasma Theory (Academic, New York, 1972).

[35] T. Kawahara, Phys. Soc. Jpn. 33, 260 (1972).

[36] V. Yu. Belashov and S. V. Vladimirov, Solitary Waves in Dispersive Complex Media (Springer-Verlag, Berlin-Heidelberg, 2005).

[37] E. M. Lifshitz and L. P. Pitaevskii, Physical Kinetics (Pergamon, Oxford, 1981). 